

\section{Government Department Leads Successful Change Challenge: National Voluntary Health Organizations Plan for Future State}

\section{Introduction}

The last decade of this century has been a difficult one for the public sector. Budgets have been cut, programs merged or eliminated, and operational staff downsized. There have been societal changes including shifts in attitudes and values, and excessive consumerism. During the decade, the 'victim' phenomena has become a prevailing force with well organized special interest groups demanding recompense for perceived injustices. And days of universal entitlements have been under critical review, if not coming to an end. All government levels have been under increased pressure for services, yet have had fewer resources with which to respond and a less clear mandate with which to work. Statements such as "I'm from the government and I'm here to help you," are met with wry smiles as many government programs' credibility has waned. New and creative responses are required to meet the challenges of responding to these 'new realities'. This article is a report of a government department that radically altered its service delivery and pioneered an experimental program based on the latest understandings from the field of organization development.

For many years a division of Health Canada had been subsidizing small, national charitable health organizations with annual operating grants, and facilitating programs to help them spend the money effectively. Many of the organizations had become dependent on these grants for their existence, yet funding was drying up; politicians were discussing ceasing the funding of charitable organizations in the volunteer sector. The new program created by the department broke out of the old mold by joining with 
the private sector to plan and operate a leadership development and organization renewal program designed to empower volunteer organizations to take charge of their well-being and manage the adjustment dictated by 'new realities'. The program was also a breakthrough to new directions for the department in that it was:

- a working model of a low cost training program organized and staffed by volunteers;

- a process oriented, learning-by-doing program that had the participants working on a change management program for their organization rather than listening to lectures and seeing how-to videos;

- organized by and with participants based on an analysis of their organization and their personal and professional interests;

- built on and evaluated by a series of measurable performance outcomes over a two year period;

- a marvelous example of successful collaboration between the public and private sectors with over eighty percent of the participating organizations achieving their measurable outcomes.

This article describes the components of the two year OD training program on strategic planning, managing change and organizational effectiveness, developed to facilitate concerns of revitalization, stabilization and institutionalization within Canadian voluntary health care organizations. The educational goals, advance preparation and residential program are reviewed. The program's planning process and unique role of the resource facilitators are described. The voluntary organizations' action plans, progress reports, and evaluations of the program's residential component are also discussed. Suggestions for next steps and the educational consultants' learnings in conducting this type of training program are highlighted. 


\section{Program Participation}

Training programs used to facilitate change frequently are designed to model series of university course modules, and are usually ends in themselves with little or no follow-up. These types of training programs rarely change participants' behaviours or impact work culture. The kind of training required to sustain change is an ongoing process and not just a program.

The training program discussed in this article, co-sponsored by the Kidney Foundation of Canada and Health Canada, is based on the Intervention and Collaborative Change Model (Dimock, 1993). It is a senior level training and development initiative on strategic planning, managing change and organizational effectiveness for approximately 22 Canadian voluntary health care organizations (approximately 70 participants). It is specifically designed for small to medium-sized organizations in the charitable health sector or closely related social service field. The program focuses on developing the knowledge, skills and leadership capacities of senior volunteers and staff to enable them to facilitate positive strategic planning and organizational renewal initiatives such that their organization can thrive and lead within the realities of the late 1990s. It provides direct planning support to the organizations involved, along with a unique personal development opportunity for their selected volunteers and staff. Each organization selects a team of three persons charged by their Board of Directors to provide leadership for charting the organization's future course. The training program was originally designed as a one-year program; due to its usefulness for most of the 22 participating organizations, Health Canada agreed to 
finance the program for a second year, and The Kidney Foundation of Canada agreed to continue as co-sponsor and manager of the program.

\section{Educational Orientation}

To provide readers with a context for the training program, some theoretical underpinnings in the field of organization development (OD) and fully discussed in Devine and Dimock, 1996, are briefly reviewed. Although the specific format of the training design as an OD strategy came out of experiences in organizational consultations and research, principles supporting it are old, traditional concepts well known in the OD field.

The action-research model, developed by Kurt Lewin (1952) and discussed by others, e.g. French and Bell (1995) typically focuses on an immediate problem in a specific local setting and tries to understand the factors affecting the problem in order to facilitate remedial planningas well as provide training for people working with the problem. The early Hawthorne studies (Roethlisberger and Dickson, 1939) demonstrate the effectiveness of involving workers in the planning of change. Marrow and French (1945), in related research dealing with change, demonstrate that if people are presented with specific information by an outsider, they are not likely to use the information even if implications are clear and related to workers' concerns. But if the information is collected by the workers, it is more likely to be put to use. Consulting those affected by a proposed change and actively involving them in decision-making has a powerful impact on behavioral outcomes. The combination of these two concepts (employee involvement and employer information) produces the underpinnings for 
making organizational personnel equal members in planning for change, and designing programs around participants' stated needs and objectives.

It is known that the social system, i.e. the community, work group or organization, is the focus of change. Groups set standards concerning members' work practices, and the more cohesive the group, the more powerful are pressures toward maintaining group norms. Changes in behaviour or work practices are most likely to be accomplished by changing the group's norms or standards (Lewin, 1952, Burke, 1994).

A social system is more than the sum of its parts; changing one part creates a dissonance within the whole, and if the whole does not change, the part will be pressured to discontinue rocking the boat (Cartwright, 1951). And, of course, the power people in the system must support the change or it has little chance of success. Change starts at the top, and while the power person cannot bring about change unilaterally, power can prevent the change of group norms especially if the power person has an administrative capacity and can control areas such as budget allocations.

Current adult education concepts also form a base for the philosophical underpinnings. Participants take responsibility for their own learning, the learning is self-directed and is experiential (learning by doing) in format. There is extensive involvement of participants in planning learning activities, and in evaluating the experience..

In summary, the educational orientation of the training program is based on sound OD and adult education principles, and on the integration of the training experience 
with the ongoing development of the participants and their organizations. This approach contrasts sharply with other training and educational programs designed as ends in themselves, and that do not necessarily develop leadership, build commitment, or have a significant impact on the work culture of their organization.

\section{The Planning Process}

A preliminary letter describing the program was sent to national volunteer organizations that met the criteria discussed above. Based on the large number of interested organizations, selection of participating organizations was based on commitment to participate in all aspects of the program, and was limited to the numbers that could be accommodated within the program budget. Selected organizations were then sent a registration package which included a registration form, an organization application and agreement form, a participant's agreement form, copies of a personal and professional interest survey, and a preliminary organizational analysis form for each member of the organization team The surveys were used to plan the design of the residential program. Participants were also sent some advance reading on planned change and organization development before the residential training component of the program.

A 10 person volunteer Program Steering Committee with representation from the national volunteer organizations in the health sector, the co-sponsors of the program, and the educational consultant guided the program development throughout. The committee planned, coordinated and evaluated the program. The personal and organization scans and surveys completed by each participant were summarized for use 
by the committee in planning the residential program's content and in forming workgroups at the residential program. The team also worked directly with additional expert resources who were brought in to assist at different phases of the program to ensure that there was access to the best knowledge and methods.

\section{The Residential Training Component}

A two and a half day training program was held, over a weekend, in a convenient residential setting. Specific content was based on analysis of participants' needs and interests, and their organizations. Particular emphasis was placed on development of specific back-home organizational improvement projects. A unique feature to be described below was the beginning of a consultation and support system for participants that continued throughout the implementation phase of the projects. A major goal of the program was to have organizational teams leave with a clear blueprint of their next steps in implementing their specific organizational change initiatives which were created at the workshop and fine tuned with the help of their colleagues and the consulting team at the residential program.

The second residential training program (one year later) was similar to the first with the focus being the organization's actual planning for change. The extensive information available from Progress Reports and Resource Facilitators' contacts with their organizations (see below) made it much easier to pinpoint the areas where help would likely be most useful. The Steering Committee was able, with confidence, to set the theme of this program as "the challenges of implementing change". We knew the 
measurable outcomes each organization had achieved during the first year and what the blockages and resistances were to each change project.

Two major changes were made in the second residential program design. A day was added to provide a 'Seminars of Excellence" series using participants' resources to demonstrate or report on outstanding successful practices. Topics included volunteer development, fund-raising and national organization cohesion. The 'how to do it' lectures of the general sessions of the first residential program were discarded, and necessary or useful input was moved to the work groups (three teams of three people), and assigned to the work group's Resource Facilitator.

The most powerful part of the residential programs was the peer review and coaching as each team reviewed its progress over the year with work group colleagues and presented its future action plans. Resource Facilitators added greatly to this experience by providing the structure where each group rotated in the role of clients (presenting their experiences and plans), the role of consultants helping the client team, and observers critiquing the process and helpfulness of the consultants. Many participants saw this aspect of the program as the best leadership training of the entire Change Challenge experience.

\section{Role of the Resource Facilitators}

A feature of the residential program worth discussion was the unique role of Resource Facilitators (a volunteer group of eight people from education, management consultants, health care and social service agencies) who acted as both trainers during the residential program and as coaches to the teams back home. This group provided 
general support and consultation as required over the next nine months. This aspect was conducted mainly by telephone with a few face-to-face meetings set up as required,

Each Resource Facilitator was assigned a workgroup composed of designated teams for three of the identified organizations and worked with the group during the residential workshops and followed up with them in the project's application phase . This represented an eight to ten day commitment. The group of Resource Facilitators acted as trainers, guiding the planning tasks and facilitating group process and relationship building. during the residential program and as coaches, supporting and consulting on each team's initiative in the back home settings as required. Conference calls and telephone check-ins were periodically conducted and reported to the educational consultant. Specific tasks of the Resource Facilitator group included structuring appropriate team building activities within the work group, facilitating the group's process with an eye on climate, involvement and participation, promoting networking between work group members and others with similar interests, acting as a planning and managing change consultant, and generally acting as the interface between the participants and the overall program, including-program content, informal and social activities, steering committee, and presenters in the general sessions of the first residential program.

\section{Application Projects and Follow-up}

During the twelve months between residential workshops and four to six months after the second residential program, triad teams continued to work on their 
organization development project with help from resource people as targeted at the residential programs. This included networking, pairing with one or more local organizations that were able to provide useful consultation and support, and periodic phone calls from the Resource Facilitators. All teams did a progress report about halfway between the residential workshops and halfway after the second workshop, for local use and to assist the planning committee in considering next steps. The teams' progress reports also served to provide outcome results from the organizations and an assessment of overall impact of the initiative.

\section{Program Evaluation and Research}

Dimock brought organization development training programs to Canada in 1962 and for fifteen years the Sir George Williams Center for Human Relations and Community Studies, Concordia University, Montreal, Quebec, conducted OD training. Program participants were from the not-for-profit sector and involved in leading planned change initiatives in their organizations. Until The Change Challenge, the most extensive follow-up of trainees had been on-site visits to perhaps $70 \%$ of one group four to six months after the training program. Hence this is the first time evaluation with all participants over a two year period has been accomplished. A review of OD literature has not found any research reports of long-term follow-up.

Another unique feature of this program is that $40 \%$ of the total budget for the overall program was allocated to evaluation and follow-up, in sharp contrast to programs where the training program stands alone as the entire activity. Evaluation 
focused on specific measurable achievement standards from the people participating in the overall program.

Some of the evaluation standards used for the first residential program included, having the participants return their completed Personal and Professional Interest Survey and the organizations' teams return their completed Preliminary Organization Analysis Survey; having organizations represented at the residential program deposit in writing before their departure a complete plan for starting their OD initiative or intervention; gaining participant satisfaction levels averaging 4 on a 5 point scale $(80 \%)$ in residential program evaluation and expecting $60 \%$ of identified organizations to show measurable changes related to their teams' plans and efforts as assessed and documented by the organizations, its members or clients, and resource facilitators ${ }^{1}$.

To assist participants gain some perspective on their organizational initiative plans and program staff to ascertain quality of plans, initiatives were rated on five criteria. Assessments suggested that most organizations were able to set clear, specific goals that approached achievability but initiatives were "powder puff" interventions unlikely to impact the organization's culture.

Progress Reports were collected three times during the two years from all organizations and rated on criteria including amount of clearly measurable change; credibility of the rating of change; the importance of the change to the overall health and goal achievement of the organization; and how much the program contributed to the togetherness and communication of the three person team.

\footnotetext{
${ }^{1}$ For further information or a copy of the complete report, contact Dr. I. Devine, Ryerson Polytechnic University, Toronto, Ontario, Canada, M5B 2K3 or irdevine@acs.ryerson.ca
} 
All data collection instruments were pulled together at the end of the program; tabulated and analyzed both individually by the educational consultant, and in a weekend meeting of the Resource Facilitator team. These extensive data were analyzed qualitatively:- content analysis, process analysis, force field analysis; and the method of characteristic differences. The data were then analyzed to establish possible relationships quantitatively comparing top half and bottom halves and unequal divisions using tetrachoric and chi square correlations. The purpose of the analysis was to understand as much as possible, the factors contributing to participants' learning and the demonstration of these learnings in successfully implementing an organization initiative. These understandings could help staff and others facilitate change in voluntary organizations and perhaps add to theory and practice of organization development.

\section{A Demonstration of a Low Cost Major Training Program}

The Change Challenge was essentially an all volunteer program as its small budget went to transportation and accommodation (out of pocket expenses) for participants, Steering Committee members and Resource Facilitators. All time was volunteered with the minor exception of some office assistance and token honorarium to selected resource people. Participating organizations believed they were not able to provide significant training to their Board or volunteer workers and could not manage any organization renewal because costs would be more than they could possibly manage. This program demonstrated, as we had continually proposed to them, that it could be done by calling on local resources at little or no cost. 


\section{Learnings about Facilitating Change in Publicly-Funded Voluntary Organizations}

While we have worked with over several hundred volunteer organizations in the past, we found these twenty-two very different. First, they were entirely dependent on volunteers for administration and operation, unlike most of the more traditional voluntary organizations that are administered by paid staff and partly to almost completely operated by staff. And second, these organizations were heavily to totally dependent on the Federal Government for funding.

There was not enough power and control to make things happen - change had to be truly collaborative, a concept with which the organizations had little experience. There was a pervasive belief that if you asked volunteers to do a real job, it would be too much to ask and they would leave. The dependency on government funding had reduced feelings of confidence and competence to take charge of their own operation. The program's focus was "intervention, collaboration and empowerment," but we did not appreciate when we started how right-on that was, or how long it would take to have an impact. The sharp contrast to traditional organizations with power, control and some independence was quickly evident.

Closely related to the psychological and empowerment concerns were practical realities such as physical distance among members, financial constraints for long distance phone calls, a scarcity of computers and internet access, and face-to-face meetings perhaps only at an Annual General Meeting. New ways of working were processed very slowly. Most organizations with their Board of Directors were stretched over a distance of more than 4,000 kilometers, and in some cases, met 
together only once a year at an annual meeting. The Change Challenge's decision to work with a three person team which included the CEO and president, contributed greatly to implementing an organization initiative. This was a dominant coalition using what power there was in the agency. The program also provided an opportunity for this coalition to meet for three or four days, a luxury many organizations had not previously experienced.

In summary 19 of 22 organizations who joined The Change Challenge achieved an identifiable level of change in their organization initiative and thus demonstrated an understanding of the data based, participatory planning method and how to use it in practice. The highlights of the program were its experiential process orientation, and its development with the involvement of the participants. The greatest learnings from the program were around the unique realities of the participating organizations and the significant role of the eighteen-month follow-up program assisted by the Resource Facilitators. The addition of Phase Two more than doubled the learnings and empowerment from the accomplishments related to the program including the team initiative and networking experiences. The greatest challenge in the program was maintaining the integrity of the adult education, experiential learning design when confronted with participants wanting to be told the answers to their concerns. A community member stated that they arrived wanting "the sage on stage" and left appreciating "the guide at their side". The self identified goals of the organizational team often fell short of the program staff's expectation and conscientiously suppressing tendencies to teach what they ought to learn in favor of helping them learn for 
themselves created both discomfort and tension in maintaining the integrity of the experiential model of learning.

\section{Learnings About Public Management of Change}

Changing the 'usual ways of doing things'was as difficult for the public department as it was for the client organizations the department was trying to help. This was a very different program for the Health Department to sponsor as previous programs were designed to tell their subsidized organizations what they should be doing to maximize their organization effectiveness and contribution to society. The differences required a role and attitude change from authoritative telling and managing an extensive grants application and review program (providing subsidies) to collaborative work facilitating the empowerment and independence of the client organizations. Such change does not happen quickly or easily and the department in this case study struggled with this issue.

After the government subsidies were withdrawn and the public department reorganized from the cutbacks, the hope was to have funding renewed in order to return to the 'subsidizing clients way of working.' While there was no commitment or enthusiasm to use any available funds to support the continued development and empowerment of these organizations, there was a strong interest in sponsoring similar training programs as the impact of the evaluation results had been so impressive. Clearly, the impact of sponsoring organization development training for charities in the voluntary sector produces the same cultural change dynamics for the public department 
as for the community serving groups. In our case, this change has started in a small, but important way.

\section{Summary and Conclusions}

Voluntary organizations are undergoing transformation in order to survive and remain vital. Planned change efforts must include strategies for systematic organization renewal rather than group or individual renewal. The program presented in this report demonstrates one way of integrating personal and professional development goals with organizational needs. An all volunteer OD training program with 22 Canadian national health organization was sufficiently successful in its first year that the Federal Government (Health Canada) asked it to continue for another year. This extension provided the opportunity to work with and follow-up the implementation of these agencies' change initiatives. The rigorous evaluation of the measurable outcomes of the two year training program provided new insights into the consulting process of facilitating change with voluntary sector organizations. 


\section{REFERENCES}

Burke, W.W. (1994). Organization development: Approaches to changing and learning. Reading, Mass: Addison-Wesley Publishing Co.

Cartwright, D. (1951). Achieving Change in People, Human Relations, 14:381392

Devine, I. and H.G. Dimock (1996). OD in Voluntary Organizations: A Training Program for National Health Organizations, Organization Development Journal, Vol. 14, No. 3, 1996, 62-70.

Dimock, H.G. and I. Devine (1995). Training for Planned Change (3rd edition). North York, Ont. Captus Press, Inc.

Dimock, H.G. (1993). Intervention and collaboration: Helping organizations to change. San Diego: Pfeiffer and Company.

Dimock, H.G. (1976). A Study of Process Oriented, Qualitative Research using Community Collaboration as a Social Action and Assessment Method of Community Development, Report Number 15. Montreal: Centre for Human Relations and Community Studies, Concordia University.

French, W. and C. Bell (1995). Organization development: Behavioral science interventions for organization improvement. 5th ed, Engelwood Cliffs, N.J., PrenticeHall

Lewin, K. (1952). Group Decision and Social Change in G. Swanson, T. Newcomb and E. Hartley (eds.). Readings in Social Psychology (2nd ed.) New York, Holt.

Marrow, A. and J.P. French Jr. (1945). Changing a Stereotype in Industry. Journal of Social Issues, 2, 33-37.

Roethlisberger, F.J. and W.J. Dickson (1939). Management and the Worker. Cambridge, Mass; Harvard University Press. 